- Technical Paper-

\title{
Application of Cyclic Magnetammetry to the Analysis of Electrochemical Reaction in a High Magnetic Field
}

\author{
Atushi Sugryama, ${ }^{a, b}, *$ Shigeyoshi MorisaKI, ${ }^{a}$ Iwao MogI, ${ }^{c}$ \\ and Ryoichi AOGAKI ${ }^{b}$
}

\author{
aDepartment of Industrial Chemistry, Graduate school of Engineering, Tokyo Metropolitan University (1-1, Minami- \\ Ohsawa, Hachiouji, Tokyo 192-0397, Japan) \\ bNational Research Laboratory for Magnetic Science, Japan Science and Technology Corporation (1-1-56, Shibashimo, \\ Kawaguchi, Saitama 333-0848, Japan) \\ cInstitute of Material Research, Tohoku University (2-1-1, Katahira, Aoba-ku, Sendai 980-0812, Japan)
}

Received January 27, 2000 ; Accepted June 14, 2000

\begin{abstract}
The cyclic-magnetammetry (CM) to analyze electrochemical reactions in a magnetic field has been newly proposed ; a periodically changing magnetic field is applied to a magnetohydrodynamic electrode (MHDE) in a vessel filled with an electrolyte solution. During the electrolysis under potentiostatic condition, current response is measured against magnetic flux density. Using the locus of the current against the magnetic flux density (the cyclic magnetammogram), we can examine the special magnetic field effects on the reaction and diffusion processes excluding the usual MHD effect. Experiments were carried out for redox reactions between ferricyanide and ferrocyanide, and copper reduction in sulfuric acid. It turned out that, in the former redox reaction, the locus converged to a straight line as expected. However, in the copper reduction, obvious hysteresis effect on magnetic field and periodic current response were observed.
\end{abstract}

Key Words : Magnetic Effect, Redox Reaction, Copper Electrodeposition, Cyclicmagnetammetry

\section{Introduction}

In recent years, strong electromagnets have become popular, facilitated by the recent development of liquid helium-free superconducting magnets, and allowed us to easily utilize magnetic fields in the range of $10 \mathrm{~T}$. Consequently, studies concerning magnetic field effects on various phenomena have been accelerated. ${ }^{1-11}$ )

Magnetic field effects on living organisms, especially on biochemical reactions have been one of the major subjects in interest. ${ }^{12-15)}$ However, the phenomena themselves have been controversial. The reason can be partly ascribed to the complexity of biochemical reactions and the difficulty in the reproducibility of experiment. We, therefore, as the first step for the investigation of biochemical reactions, paid attention to simpler reactions, i.e., electrochemical reactions, which play an important role in biochemical process.

In an electrochemical system, an externally applied magnetic field yields a solution flow induced by the Lorentz force to enhance the mass transfer process, which is well known as the magnetohydrodynamic (MHD) effect. ${ }^{16-20)}$ On detecting the effect on electron transfer processes, a difficult problem is raised that the MHD effect is inevitably involved in electrode reactions under the external magnetic field. ${ }^{21-27)}$ Therefore, even if we observe some magnetic field effect on electrolytic current, it can not be directly identified with the effect on electron transfer processes. At least, a part is attributable to the change in diffusion process, i.e., the MHD effect. So far there have not been any methods available to dis- criminate the electron transfer from the MHD effect. In stead of suppressing the effect, we rather attempted to positively employ the MHD effect to separate only electron transfer process from overall reactions. ${ }^{28,29)}$

In this study, a new type of electrode, i.e., the MHD electrode (MHDE) was used to analyze the electron transfer process in copper reduction in the magnetic field Copper reduction has often been examined in the magnetic field; ${ }^{22}$ many of them have so far reported that no special effect except for the MHD effect can be found. However, remarkable reduction of the reaction rate together with the hysteresis effect was observed, ${ }^{29)}$ so that it was speculated that this phenomenon came from the decrease of active points on the copper crystal surfaces. However, the details have not yet been clarified.

Therefore, to examine such magnetic field effects more precisely, we have newly developed an electrochemical method called the "cyclic magnetammetry (CM)," in which by periodically sweeping magnetic flux density with time under potentiostatic condition the response of electrolytic current is measured to examine the electron transfer and diffusion processes.

In this paper, the details of the theoretical bases of the $\mathrm{CM}$ are described. Then, its application to copper reduction is examined in comparison with a ferricyanide/ferrocyanide system.

\section{Theoretical}

The schematic configuration of MHDE is shown in Fig. 1. It is immersed in a large vessel filled with an elec- 


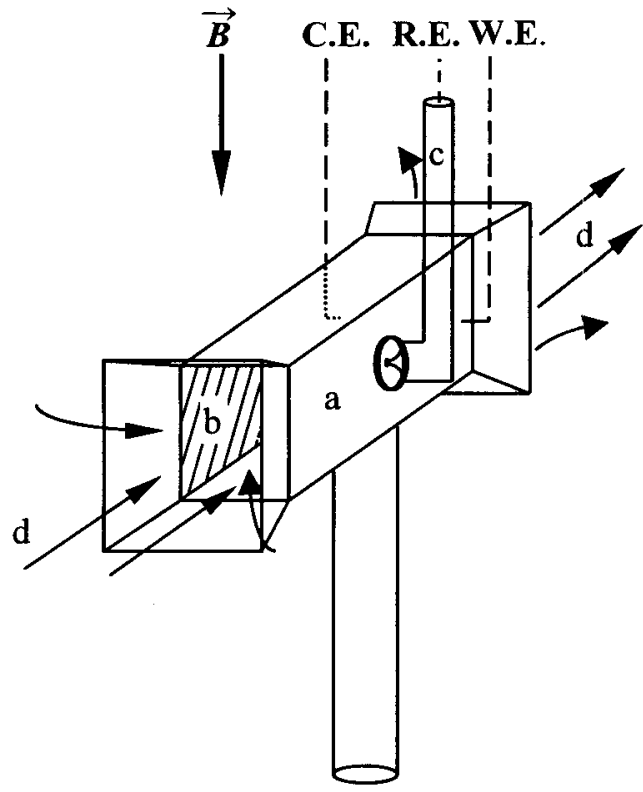

Fig. 1 Schematic configuration of MHDE (magnetohydrodynamic electrode). a, working electrode ; b, counter electrode ; c, Luggin capillary ; $d$, stream line ; $B$, external magnetic flux density.

trolyte solution, the working and counter electrodes are set face to face on the inner walls of a rectangular channel with two open ends. A reference electrode is inserted from behind the working electrode not to disturb the solution flow in the channel.

When an overpotential is applied to the working electrode, a current flows between the working and counter electrodes. The magnetic field is, as shown in Fig. 2, vertically imposed to the electrolytic current, so that the Lorentz force is induced and the electrolyte solution starts to move to form a laminar boundary layer flow. The flow is consisted of two regions, the main flow and the thin boundary layer as shown in Fig. 2, because the channel length is short $(l=2 \mathrm{~cm})$ which is almost equivalent to the channel width $(w=1 \mathrm{~cm})$ and the Reynolds number is smaller than 2000 . The magnetic Reynolds number, $R_{\mathrm{m}}=\mu \sigma U l$ can be estimated with the quantities, magnetic permeability $\mu \approx \mu_{0}=4 \pi \times 10^{-7} \mathrm{H} \mathrm{m}^{-1}$, electrical conductivity $\sigma<50 \mathrm{~S} \mathrm{~m}^{-1}$, the length of channel $l=2 \mathrm{~cm}$ and the velocity of main flow $U<10 \mathrm{~cm} \mathrm{~s}^{-1}$; hence the magnetic Reynolds number $R_{\mathrm{m}}$ is about $10^{-7}$, which means that the induced magnetic field can be neglected.

This motion of the solution enhances the mass transfer of the active ionic species in the solution. If the sweep rate of the magnetic flux density is sufficiently small, the average current density measured is consistent with the steady diffusion current density, whose function form has already been given as $^{17)}$

$$
\bar{i}= \pm H^{*}\left(C_{0}-C_{\mathrm{S}}\right)^{4 / 3} B^{1 / 3}
$$

where " \pm " correspond to anodic and cathodic reactions, respectively. Then, $C_{0}$ and $C_{\mathrm{S}}$ designate the bulk and surface concentrations of the active ionic species, respec- tively. $B$ is the external magnetic flux density and $H^{*}$ is the coefficient expressed as,

$$
\begin{aligned}
H^{*}= & 0.753(n F D)^{4 / 3}(v / D)^{4 / 9} v^{-2 / 3} \rho^{-1 / 3} \gamma^{1 / 3} \\
& \left(\sqrt{X^{2}}-\sqrt{X_{1}}\right)^{4 / 3}\left(X_{2}-X_{1}\right)^{-1}
\end{aligned}
$$

where $n$ the charge number participating reaction, $F$ Faraday constant, $D$ the diffusion coefficient, $v$ the kinematic viscosity, and $\rho$ is the density. $X_{1}$ and $X_{2}$ are the edge positions of the electrode on the upper and lower parts of the stream, respectively.

As the absolute value of the overpotential increases, the reaction processes are activated, so that the supply of the active ionic species from the bulk solution does not keep up with the consumption by the reactions on the electrode surface. In this situation, the diffusion current density reaches a limiting state corresponding to $C_{\mathrm{S}}=0$ in Eq. (1), i.e., the limiting diffusion current density $\bar{i}_{\mathrm{L}}$ emerges. From Eq. (1), it follows that

$$
\bar{i}_{\mathrm{L}}= \pm H^{*} C_{0}^{4 / 3} B^{1 / 3} .
$$

Taking the ratio of Eq. (1) to Eq. (3), we obtain

$$
\bar{i} / \overline{i_{\mathrm{L}}}=\left\{\left(C_{0}-C_{\mathrm{S}}\right) / C_{0}\right\}^{4 / 3} .
$$

If the electrode reaction is considered as the first order reaction with regard to the surface concentration of active species, the average reaction current density is

$$
\bar{i}= \pm n F k C_{s}
$$

where $k$ is the overall reaction-rate coefficient. Actually, in the case of cathodic reaction,

$$
k=k_{0} \exp \left(-\alpha_{\mathrm{c}} F \Delta \Phi / R T\right)
$$

in the case of anodic reaction,

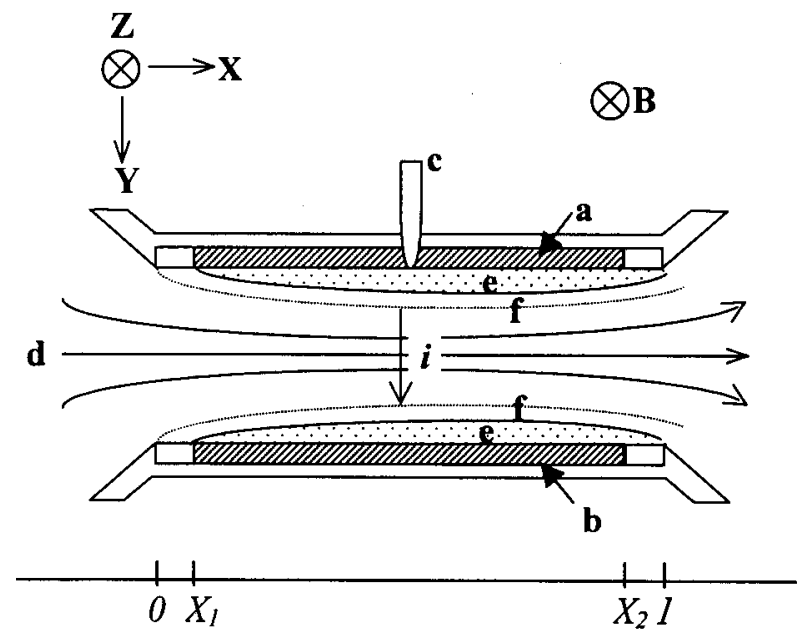

Fig. 2 Schematic figure of boundary layers and diffusion layers formed along electrodes. a, working electrode ; b, counter electrode ; c, Luggin capillary ; d, stream line ; e, diffusion layer ; $\mathrm{f}$, boundary layer ; $i$, current density ; $B$, external magnetic flux density. 


$$
k=k_{0} \exp \left(-\alpha_{\mathrm{a}} F \Delta \Phi / R T\right)
$$

where $\alpha_{\mathrm{c}}$ and $\alpha_{\mathrm{a}}$ are the transfer coefficients for cathodic and anodic reactions, respectively. $R$ is the universal gas constant, and $T$ is the absolute temperature. Then, $\Delta \Phi$ implies the activation overpotential measured from the equilibrium potential, and $k_{0}$ is the reaction rate constant at the equilibrium potential $(\Delta \Phi=0)$.

From Eqs. (4) and (5),

$$
\bar{i}= \pm n F k C_{0}\left\{1-\left(\bar{i} / \overline{i_{\mathrm{L}}}\right)^{3 / 4}\right\}
$$

is derived. Hence, as the ultimate reaction current density $\bar{i}_{\mathrm{k}}$, we define the following equation,

$$
\bar{i}_{\mathrm{k}}= \pm n F k C_{0} \text {. }
$$

Then, substitution of Eqs. (3) and (9) into Eq. (8) leads to the eventual equation,

$$
1 /|\bar{i}|-1 /\left|\bar{i}_{\mathrm{k}}\right|=1 /\left\{H^{* 4 / 3} C_{0}(B|\bar{i}|)^{1 / 4}\right\} .
$$

Therefore, if we sweep the magnetic flux density at a sufficiently low rate, it is expected that the $1 /|\bar{i}|$ vs. $1 /(B|\vec{i}|)^{1 / 4}$ plot, expressed by Eq. (10), can be automatically drawn by recording the transient of the current response (i.e., the cyclic magnetammogram). Two kinds of sweep modes are considered; steady and non-steady modes as shown in Figs. 3 and 4.

In the steady mode (Fig. 3), after measuring a steady current density at a certain magnetic flux density, the magnetic flux density is changed to the next value, then another steady current is measured. This procedure is repeated until the locus, i.e., the cyclic magnetammogram is completed. The sweep mode thus becomes step-

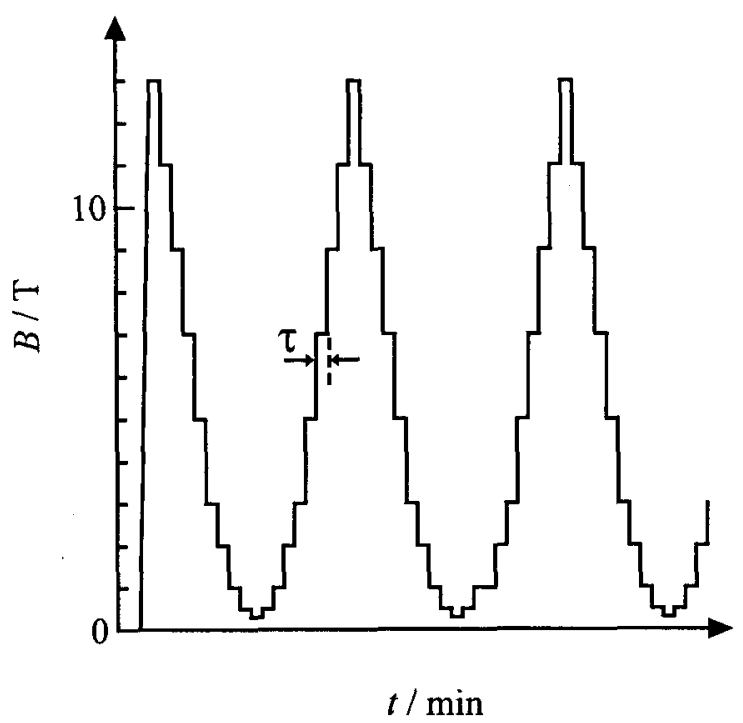

Fig. 3 Sweep mode of magnetic field strength (steady state mode). $\tau$ is the retention period of the constant magnetic flux density until the current value reaches the steady state, which is changed by the degree of the relaxation of the reaction system. In case of the copper reduction, $\tau$ was 1 minute or 3 minutes. wise. On the other hand, in nonsteady mode, the sweep form of the magnetic flux density is, as shown in Fig. 4, continuous in a triangular shape. In this case, except for the ultimately slow scan, the current observed does not take steady state but nonsteady state.

Therefore, in the absence of the special magnetic field effects concerning reaction and diffusion processes, the MHD effect should provide a locus of the nonsteady mode converging to that of the steady mode as the sweep rate decreases. This provides us the means to examine the hysteresis effect with the magnetic field by changing the sweep rate.

\section{Experimental}

Two different experiments were performed; one was the redox reaction for a ferricyanide/ferrocyanide system based on the hypothesis that it should provide an ordinal straight line described by Eq. (10). The other was the copper reduction for a copper/copper sulfate system that was carried out to apply the $\mathrm{CM}$ to analyze the hysteresis effect on the copper reduction.

MHDE was composed of a rectangular channel with two open ends, which was $1 \mathrm{~cm}$ height and $0.5 \mathrm{~cm}$ width. The working and counter electrodes having $1 \times 2 \mathrm{~cm}^{2}$ area were made of platinum plates for the redox reaction and copper plates for the copper reduction, respectively. $\mathrm{An} \mathrm{Ag} / \mathrm{AgCl}$ electrode was employed as a reference electrode, which was set as mentioned before, from behind the working electrode through a Luggin capillary tip filled with agar-agar gel saturated by $\mathrm{KCl}$.

Before experiments of the redox reaction, the working electrode was activated by the cyclic voltammetry in a different vessel filled with $10^{3} \mathrm{~mol} \mathrm{~m}^{-3} \mathrm{H}_{2} \mathrm{SO}_{4}$ solution. After ascertaining constant activity of the working electrode, the MHDE was purified by twice-distilled water, and then transferred to the vessel filled with a solution having $\mathrm{K}_{4}\left[\mathrm{Fe}(\mathrm{CN})_{6}\right]$ and $\mathrm{K}_{3}\left[\mathrm{Fe}(\mathrm{CN})_{6}\right]+10^{3} \mathrm{~mol} \mathrm{~m}^{-3} \mathrm{KCl}$ as a supporting electrolyte.

For copper reduction, the electrode surface was in-

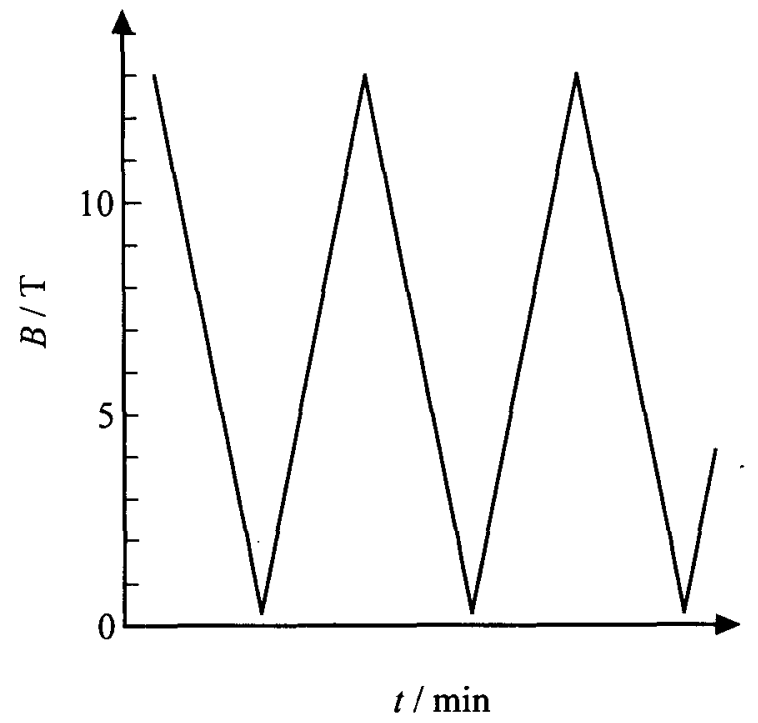

Fig. 4 Sweep mode of magnetic field strength (nonsteady state mode). 
itially polished by alumina-polishing papers with grain sizes down to $1 \mu \mathrm{m}$. After treating the MHDE with twicedistilled water in a supersonic washer, the cell was also transferred to a vessel filled with $\mathrm{CuSO}_{4}+10^{3} \mathrm{~mol} \mathrm{~m}{ }^{-3}$ $\mathrm{H}_{2} \mathrm{SO}_{4}$ as a supporting electrolyte.

At the beginning, the solution was deaerated by bubbling argon gas for more than 20 minutes. During experiments, to prevent the solution from the contamination of oxygen, argon gas was introduced into the vessel, flowing above the solution surface. Temperature was kept at $10 \pm 1^{\circ} \mathrm{C}$.

Measurements were carried out as follows; at first, the magnetic flux density was adjusted at the initial value. Then, with the electrolysis under potentiostatic condition regulated by a potentiostat (Potentio-Galvanostat 2000, Toho Technical Research), the magnetic flux density was cyclically swept in a given rate. At the same time, the current response was recorded by an X-Y recorder (F-

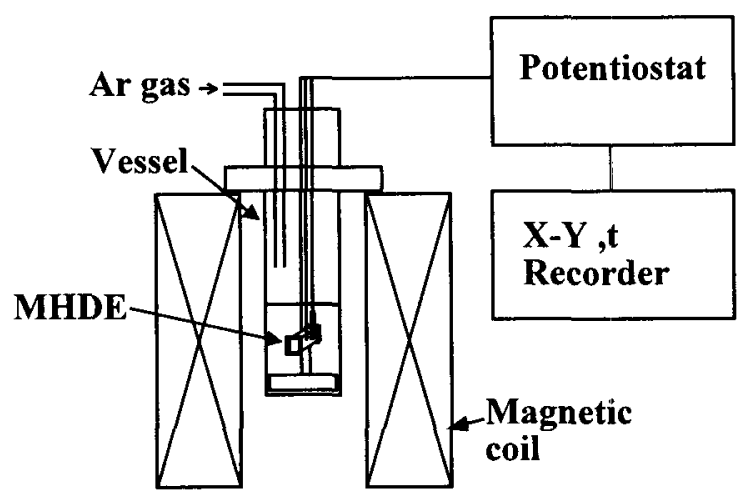

Fig. 5 Block diagram of apparatus.

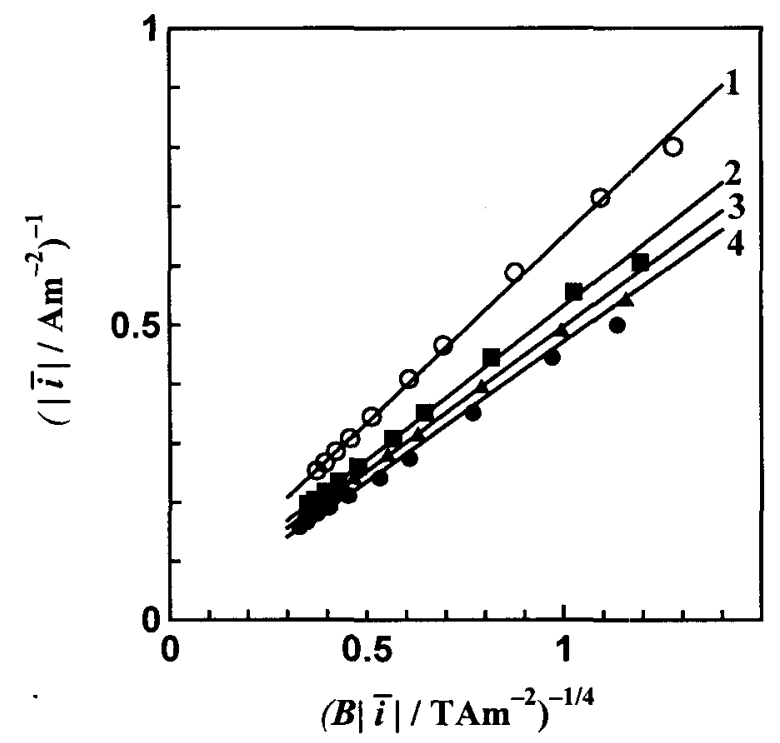

Fig. 6 Steady cyclic magnetammograms for ferricyanide reduction. $\left[\mathrm{K}_{3}\left[\mathrm{Fe}(\mathrm{CN})_{6}\right]\right], 10 \mathrm{~mol} \mathrm{~m}^{-3} ;\left[\mathrm{K}_{4}\left[\mathrm{Fe}(\mathrm{CN})_{6}\right]\right]$, $10 \mathrm{~mol} \mathrm{~m}^{-3}$; [KCl], $1000 \mathrm{~mol} \mathrm{~m}^{-3}$. Temperature is $10 \pm 1{ }^{\circ} \mathrm{C}$, and applied overpotentials are $1,-40 \mathrm{mV} ; 2,-60 \mathrm{mV} ; 3$, $-80 \mathrm{mV} ; 4,-100 \mathrm{mV}$. Each extrapolation value of straight line to the ordinate gives the reciprocal of the average reaction current density.
$42 \mathrm{P}$, Riken Electronics). Figure 5 shows the schematic block diagram. The magnet used was the water-cooled resistive magnet (WM-5) in High Field Laboratory for Superconducting Materials, Institute for Material Research, Tohoku University. The magnetic flux density was changed between $0.3 \mathrm{~T}$ and $13 \mathrm{~T}$.

\section{Results and Discussion}

The redox reaction in the present case is written by the following reversible reaction,

$$
\mathrm{Fe}(\mathrm{CN})_{6}{ }^{4-} \rightleftarrows \mathrm{Fe}(\mathrm{CN})_{6}{ }^{3-}+\mathrm{e}
$$

To verify the validity of Eq. (10) in experiments, we measured the steady currents of ferricyanide reduction against various magnetic flux densities under potentiostatic condition. Then, plotting $1 /|\bar{i}|$ vs. $1 /(B|\bar{i}|)^{1 / 4}$ as shown in Fig. 6, we obtained a steady cyclic magnetammogram for ferricyanide reduction with various overpotentials.

All the data agreed in providing a straight line. From Eq. (10), the slope is expressed as $H^{*-3 / 4}$ and the extrapolation to the ordinate gives $1 /\left|\bar{i}_{k}\right|$. Therefore, we can calculate the diffusion coefficient of ferricyanide ion with Eq. (2), and the reaction rate with Eq. (9). The constant slope shown in Fig. 6 thus indicates that not only the diffusion coefficient but also the reaction rate are kept constant under the magnetic flux densities up to $13 \mathrm{~T}$. We obtained the diffusion coefficient $D=5.1 \times$ $10^{-10} \mathrm{~m}^{2} \mathrm{~s}^{-1}$ and the exchange current density $i_{0}=55 \mathrm{~A}$ $\mathrm{m}^{-2}$ from Fig. 6, whereas the experimental results in zero magnetic field with rotating disk and current pulse were $D=5.7 \times 10^{-10} \mathrm{~m}^{2} \mathrm{~s}^{-1}$ and $i_{0}=52 \mathrm{~A} \mathrm{~m}^{-2}$, which suggests that there is no effects on the reaction process in the magnetic fields at least up to $13 \mathrm{~T}$.

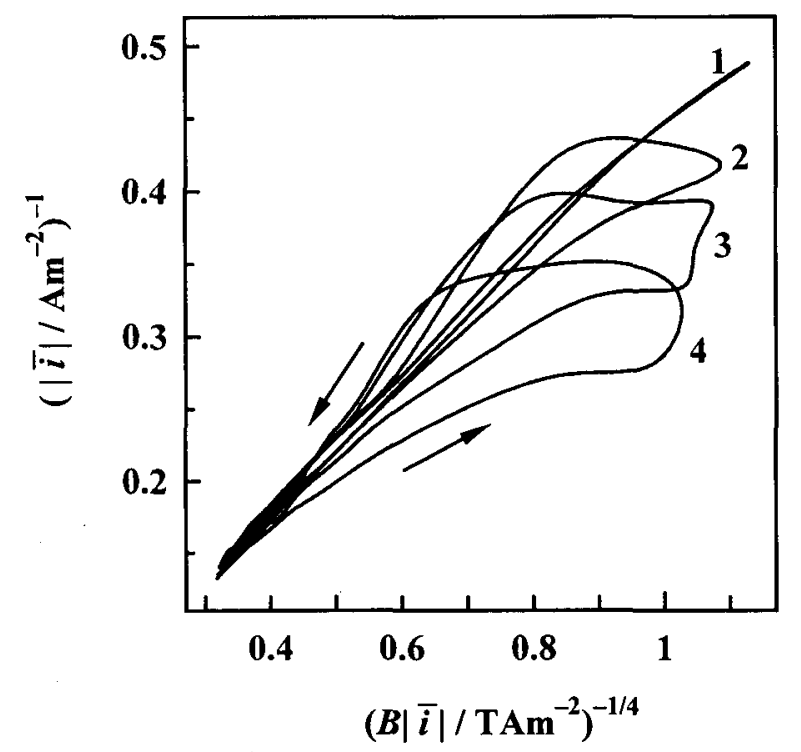

Fig. 7 Dependence of cyclicmagnetammogram on sweep rate of magnetic field. Solution composition and temperature are the same as Fig. 6, and overpotentials are $-70 \mathrm{mV}$. 1 , steady-state mode; $2,1 \mathrm{~T} \min ^{-1} ; 3,3 \mathrm{~T} \min ^{-1} ; 4,7.5 \mathrm{~T}$ $\min ^{-1}$. 
In the case of the nonsteady CM, we measure the current response against the sweeping magnetic flux density.

Then, in the same manner as the steady case shown in Fig. 6, the nonsteady magnetammogram can be drawn. Figure 7 shows the effect of the sweep rate on the locus.

All the loci form closed loops; especially at a rapid sweep rate, in the lower magnetic field region, the locus gives a largely spread loop. At the higher magnetic field region, or as the sweep rate decreases, it converges to a straight line which has already been exhibited in Fig. 6 . These behaviors of the loci can be ascribed to the following two reasons; one is the retarded acceleration of the

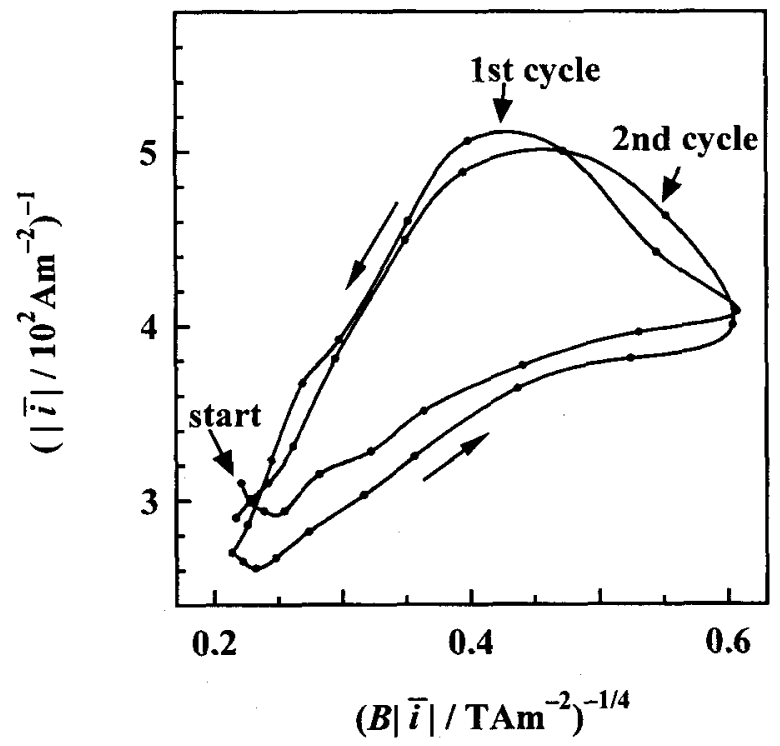

Fig. 8 Steady-state cyclic magnetammogram for copper reduction in a sulfuric acid solution. $\left[\mathrm{CuSO}_{4}\right], 300 \mathrm{~mol} \mathrm{~m}^{-3}$; $\left[\mathrm{H}_{2} \mathrm{SO}_{4}\right], 1000 \mathrm{~mol} \mathrm{~m}^{-3}$. Temperature is $10 \pm 1{ }^{\circ} \mathrm{C}$ and applied overpotential is $-110 \mathrm{mV}$.

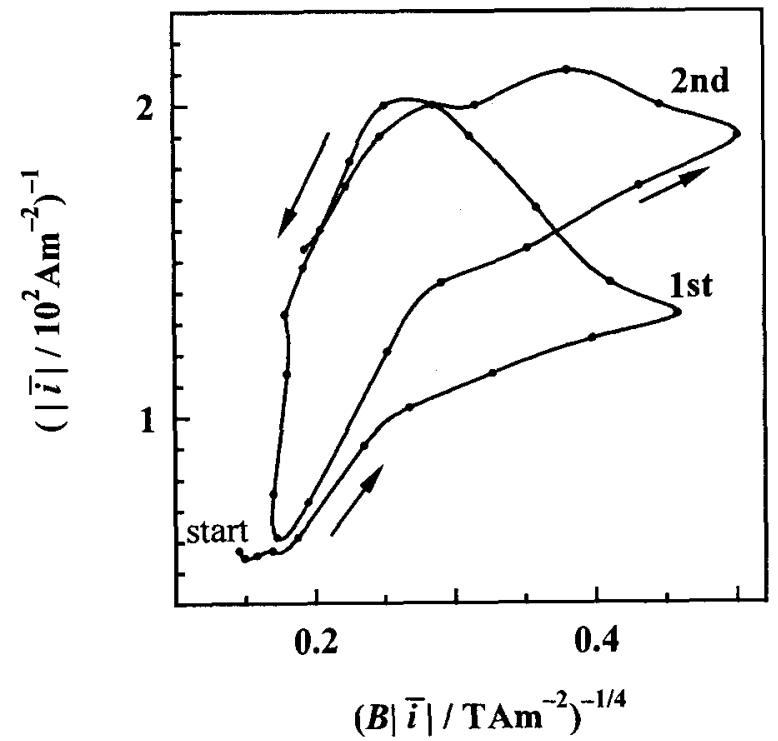

Fig. 9 Steady-state cyclic magnetammogram for copper reduction in a sulfuric acid solution. Solution composition and temperature are the same as Fig. 8. Applied overpotential is $-150 \mathrm{mV}$. velocity by the viscosity, and another is the elongated deceleration by the inertia of the MHD flow.

As mentioned in the previous papers, ${ }^{29)}$ copper reduction receives an intense magnetic field effect in the electron transfer process, i.e., a hysteresis effect in electron transfer process. To exhibit the effect more clearly, experiments in the steady mode were selected; the cyclic magnetammograms were made at the overpotentials of $-110 \mathrm{mV},-150 \mathrm{mV}$ and $-200 \mathrm{mV}$ as shown in Figs. 8 to 10 .

The copper reduction in a sulfuric acid solution is expressed in the following, ${ }^{30)}$

$$
\begin{aligned}
& \mathrm{Cu}^{2+}+\mathrm{e} \longrightarrow \mathrm{Cu}^{+} \\
& \mathrm{Cu}{ }^{+}+\mathrm{e} \longrightarrow \mathrm{Cu}
\end{aligned}
$$

Here, the first reaction in Eq. (12) takes the rate determining step, so that the reaction can be treated as the first order reaction with regard to the surface concentration of $\mathrm{Cu}^{2+}$ ion. Therefore, if there is no special magnetic field effects, as mentioned in Fig. 6, the steady cyclic magnetammogram is expected to give a single straight line. However, as shown in Figs. 8 to 10 , the actual situation was quite different; at the lower overpotential (Fig. 8), the locus formed an expanding loop, which did not converge to a straight line even in the range of the higher magnetic field. With increasing overpotential, such loop shifted to the higher magnetic fields, and at least two branches newly emerged (Fig. 9). At the overpotential of $200 \mathrm{mV}$ (Fig. 10), the locus converged in the lower magnetic fields, giving three different lines corresponding to different reaction rates.

This phenomenon could not be attributed to the change of surface area by the crystal growth ${ }^{31)}$ such as the dendritic growth, of which conclusion was ascertained by obtaining the cyclic magnetammogram of the

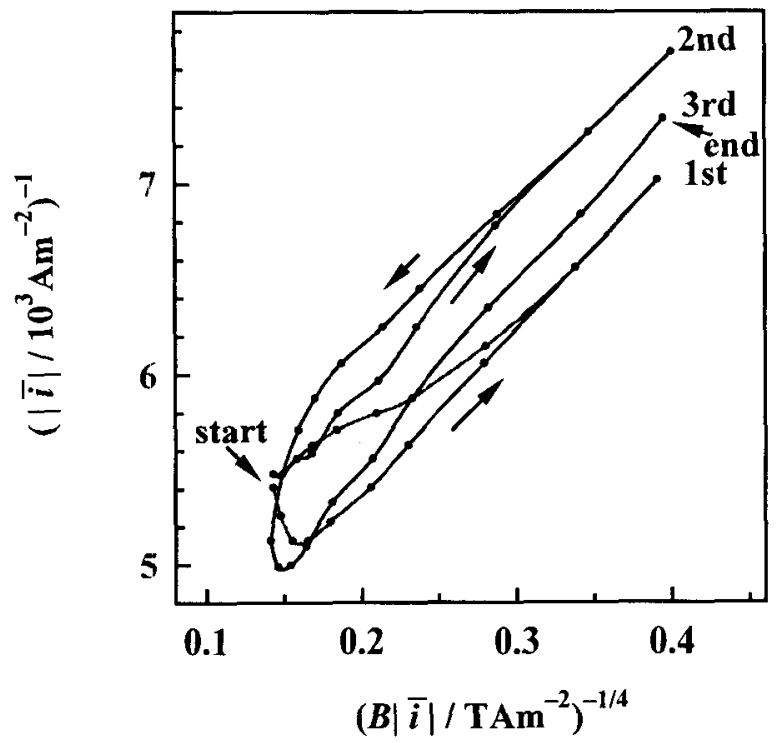

Fig. 10 Steady-state cyclic magnetammogram for copper reduction in a sulfuric acid solution. Solution composition and temperature are the same as Fig. 8. Overpotential is $-200 \mathrm{mV}$. 
same copper reduction in the nonsteady mode. Namely, in the nonsteady mode, because of short experimental time, it is expected that the surface area does not so much change. Figure 11 represents the nonsteady cyclic magnetammograms at the overpotential of $-110 \mathrm{mV}$ in different sweep rates, $1 \mathrm{~T} \mathrm{~min}^{-1}$ and $3 \mathrm{~T} \mathrm{~min}{ }^{-1}$. As shown in Fig. 11, the reciprocal of the current density increased after every scanning in $3 \mathrm{~T} \mathrm{~min}{ }^{-1}$, indicating a continuous degeneration of the reaction activity. At the rate of $1 \mathrm{~T} \mathrm{~min}^{-1}$, a closed locus appeared being fixed at the degenerated state. In both cases, the current decreased beyond the magnetic flux density of about $2 \mathrm{~T}$. This implies that the degeneration occurs beyond $2 \mathrm{~T}$. At the same time, a flat deposit without any dendrites was observed. Figure 12 shows a SEM photo after $60 \mathrm{~min}$ utes deposition during the $\mathrm{CM}$, which is quite different from that of the surface deposited under zero magnetic field. In order to observe the effect of the mechanical flow at the same time, the electrodeposition under zero magnetic field was carried out by using a rotating disk electrode.

As stated at the beginning of this paper, the copper reduction in a high magnetic field receives the decrease of the reaction rate accompanied with the hysteresis effect. In the previous paper, ${ }^{28)}$ we clarified that there is no change in the transfer coefficient during the reduction of the copper deposition rate. According to Olivier et al., ${ }^{21)}$ in nonsteady electrochemical method such as the electrochemical impedance method, no change in the copper deposition rate was observed until $1 \mathrm{~T}$. On the other hand, from the nonequilibrium fluctuation theory, ${ }^{32-37)}$ it was concluded that the electrochemical nucleation process like the copper deposition proceeds through the unstable growth of the fluctuations. Such fluctuations are formed by the non-uniform distributions of the physical quantities, e.g., concentration and current, which are ac-

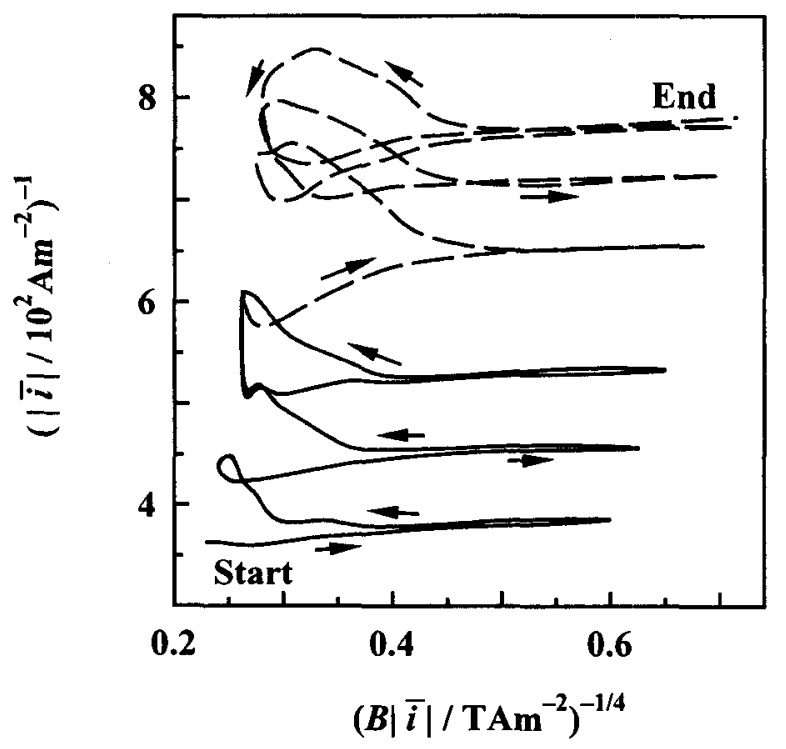

Fig. 11 Nonsteady-state cyclic magnetammo-gram for copper reduction. Sweep rates of the magnetic flux density are; solid line, $3 \mathrm{~T} \mathrm{~min}^{-1}$; dashed line, $1 \mathrm{~T} \mathrm{~min}^{-1}$. Solution composition and temperature are the same as Fig. 8. companied with heterogeneous surface reactions. All these phenomena in copper reduction are attributed to the autocatalytic process concerning such fluctuations. Though the apparent reaction rate is thus enhanced by the fluctuations, the actual reaction rate measured by nonsteady methods is not affected by the magnetic field. The MHD flow tends to hinder such unstable growth of the fluctuation because its intense stream blows off the concentration fluctuation of copper ion, so that the apparent reaction rate is suppressed to the initial low level. Moreover, this suppression is reinforced by the microMHD flow, which is induced by the interaction between the magnetic field and the current fluctuation in the diffusion layer in the vicinity of the electrode. The solution outside the diffusion layer but inside the boundary layer thus flows in a laminar mode without any disturbance. The spread part of the locus shown in Figs. 8 to 11 suggests that the fluctuations receive the micro-MHD effect from the applied magnetic field, whereas the straight part indicates that the magnetic field does not directly affect them, and only the usual MHD effect expressed in Eq. (1) is effective. The growth rate of the fluctuation tends to increase with increasing overpotential; however,
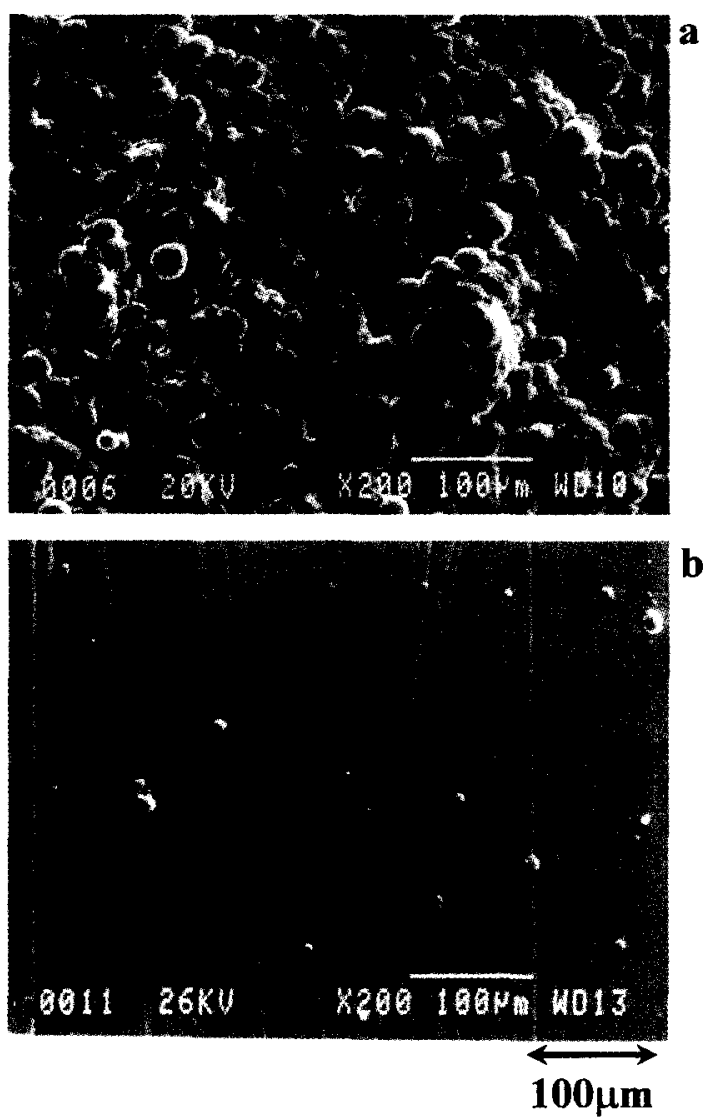

Fig. 12 SEM photos of deposited copper surfaces. (a) copper deposition onto a rotating disk electrode (rotational speed $=1500 \mathrm{rpm}$ ) under zero magnetic field; (b) copper deposition onto a MHDE by non-steady cyclicmagnetammetry. Magnetic field was swept between $0.3 \mathrm{~T}$ and $13 \mathrm{~T}$ in the rate of $0.5 \mathrm{~T} \mathrm{~min}^{-1}$. Solution composition and temperature were the same as Fig. 8. Deposition time was about 60 minutes. Applied overpotential was $-110 \mathrm{mV}$. 
the reaction process always receives the suppression especially from the micro-MHD flow. At the lower potentials, both effects are balanced with each other, i.e., sometimes the growing tendency overcomes the suppression, and sometimes the suppression effect prevails over the growing tendency, which is thought to result in the spread part. At the higher potentials, the fluctuation develops escaping from the shackles of the micro-MHD effect, and reaches the steady reaction rate by the help of the usual MHD effect, so that the straight part is obtained. From this discussion, we can also expect that such straight part is enlarged to lower magnetic fields with increasing overpotential. Therefore, we can summarize why the branches are observed in Figs. 9 and 10 as follows; in the range of the higher magnetic field (at the spread part), the nonequilibrium fluctuations are affected by the magnetic field, and the apparent reaction rate is resultantly changed. However, in the lower magnetic fields, the nonequilibrium fluctuations do not receive any interference from the magnetic field, so that the reaction rates are fixed, i.e., the branches corresponding to the multi-reaction rates appear as straight lines.

\section{Conclusions}

It was experimentally demonstrated that the cyclic magnetammetry is one of the most useful methods for analyzing the magnetic field effects in electrochemistry. From the current response against a periodically swept magnetic field strength, a kind of locus was obtained. This locus, which was named as the "cyclic magnetammogram", gave the information about the electron transfer rates and diffusion coefficients for a ferricyanide/ferrocyanide redox system; it was concluded that in the magnetic field up to $13 \mathrm{~T}$, there was no magnetic field effect in this system.

However, for copper reduction in a sulfuric acid solution, an obvious hysteresis effect on the apparent reaction rate was observed together with a remarkable decrease of the rate. Then, it was found that this reaction system contained multiple reaction states in high magnetic fields. Finally, such phenomenon was attributed to the blocking effect of the micro-MHD flow on the unstable growth of the nonequilibrium fluctuations accompanied with copper reduction.

\section{Acknowledgements}

The authors wish to express their appreciation to High Field Laboratory for Superconducting Materials, Institute for Material Research, Tohoku University for granting the use of the water-cooled resistance magnet, and also to Prof. Eiko Ito of Tokyo Metropolitan University for fruitful, encouraging discussion.

\section{References}

1) Y. Tanimoto, H. Hayashi, S. Nagakura, H. Sakuragi, and K. Tokumaru, Chem. Phys. Lett., 41, 267 (1976).

2) N. J. Turro, M. -F. Chow, C. J. Chung, and C. H. Tung, J. Am. Chem. Soc., 102, 7391 (1980).

3) K. Higashitani and J. Oshitani, J. Colloid Interface Sci., 204, 363(1998).
4) N. Hirota, T. Homma, H. Sugawara, K. Kitazawa, M. Iwasaka, S. Ueno, H.Yokoi, Y. Kakudate, S. Fujiwara, and M. Kawamura, Jpn. J. Appl. Phys., 34, L991 (1995).

5) T. Z. Fahidy, J. Appl. Electrochem., 13, 553 (1983).

6) K. W. Busch, M. A. Busch, D. H. Parker, R. E. Darling, and J. L. McAtee, Jr., Corrosion-NACE, 42, 211 (1986).

7) I. Mogi, S. Okubo, and Y. Nakagawa, J. Phys. Soc. Jpn., 60, 3200 (1991).

8) Y. Ikezoe, N.Hirota, J. Nakagawa, and K. Kitazawa, $\mathrm{Na-}$ ture, 393,749 (1998).

9) S. Yanagiya, G. Sazaki, S.D. Durbin, S. Miyashita, T. Nakada, H.Komatsu, K. Watanabe, and M. Motokawa, $J$. Cryst. Growth, 196, 319 (1999).

10) H. Kurashima and S. Ozeki, J. Surf. Sci. Soc. Jpn., 20, 777 (1999) (in Japanese).

11) A. Sato, J. Surf. Sci. Soc. Jpn., 20, 758 (1999) (in Japanese).

12) A. J. Hoff, H. Rademaker, R. van Grondelle, and L. N. M. Duysens, Biochim. Biophys. Acta, 460, 547 (1977).

13) L. M. Vainer, A. V. Podoplelov, T. V. Leshina, R. Z. Sagdeyev, and Yu. N. Molin, Biophysics, 23, 234 (1978).

14) A. J. Hoff and E. J. Lous, Biophysical Effects of Steady Magnetic Field (Eds. G. Maret, J. Kiepenhouer, and N. Boccara), Springer-Verlag, p.74 (1986).

15) T. Koana, M. Ikehata, and M. Nakagawa, Bioelectrochem. Bioenerg., 36, 95 (1995).

16) R. Aogaki, K. Fueki, and T. Mukaibo, Denki Kagaku (presently Electrochemistry), 43, 504 (1975).

17) R. Aogaki, K. Fueki, and T. Mukaibo, Denki Kagaku (presently Electrochemistry), 43, 509 (1975).

18) R. Aogaki, K. Fueki, and T. Mukaibo, Denki Kagaku (presently Electrochemistry), 44, 89 (1976).

19) R. Aogaki and K. Fueki, J. Electrochem. Soc., 131, 1295 (1984).

20) G. B. Boum and A. Alemany, Electrochim. Acta, 44, 1749 (1999).

21) O. Aaboubi, J. P. Chopart, J. Douglade, A. Olivier, C. Gabrielli, and B. Tribollet, J. Electrochem. Soc., 137, 1796 (1990).

22) J. P. Chopart, J. Douglade, P. Fricoteaux, and A. Olivier, Electrochim. Acta, 36, 459 (1991).

23) J. Lee, X. Gao, L. D. A. Hardy, and H. S. White, J. Electrochem. Soc., 142, L90 (1995).

24) S. R. Ragsdale, K. M. Grant, and H. S. White, J. Am. Chem. Soc., 120, 13461 (1998).

25) F. Takahashi, Y. Sakai, and T. Tamura, Electrochim. Acta, 25, 1147 (1983).

26) S. Mori. K. Satoh, and A. Tanimoto, Electrochim. Acta, 39, 2789 (1994).

27) K. Kim and T. Z. Fahidy, J. Electrochem. Soc., 142, 4196 (1995).

28) S. Yamanaka, R. Aogaki, M. Yamato, E. Ito, and I. Mogi, SCI. REP. RITU, Vol. 38, NO. 2, 399 (1993).

29) R. Aogaki, T. Negishi, M. Yamato, E. Ito, and I. Mogi, Physica B, 201, 611 (1994).

30) J. O'M. Bockris and A. R. Despic, Physical Chemistry Vol. IXE (Eds. H.Eyring, D. Henderson, and W. Jost), Academic Press, New York, p.627 (1970).

31) P. Fricoteaux, $\mathrm{Ph}$. D. Thesis, Univerisite de Reims, France (1991).

32) A. Tadano, M. Asanuma, and R. Aogaki, J. Cryst. Growth, 166, 1111 (1996).

33) R. Aogaki, J. Chem. Phys., 103, 8602 (1995). 
34) R. Aogaki, A. Yamada, and A. Tadano, J. Chem. Phys., 103, 8616 (1995).

35) A. Tadano and R. Aogaki, J. Chem. Phys., 106, 6126 (1997).

36) A. Tadano and R. Aogaki, J. Chem. Phys., 106, 6138 (1997).
37) A. Tadano and R. Aogaki, J. Chem. Phys., 106, 6146 (1997).

38) K. Shinohara and R. Aogaki, Electrochemistry, 67, 126 (1999).

39) M. Asanuma and R. Aogaki, to be published in IOM Communications. 